\title{
ON THE DIMENSION THEORY OF VON NEUMANN ALGEBRAS
}

\author{
DAVID SHERMAN
}

(Dedicated to the memory of Gert K. Pedersen)

\begin{abstract}
In this paper we study three aspects of $(\mathscr{P}(\mathscr{M}) / \sim)$, the set of Murray-von Neumann equivalence classes of projections in a von Neumann algebra $\mathscr{M}$. First we determine the topological structure that $(\mathscr{P}(\mathscr{M}) / \sim)$ inherits from the operator topologies on $\mathscr{M}$. Then we show that there is a version of the center-valued trace which extends the dimension function, even when $\mathscr{M}$ is not $\sigma$-finite. Finally we prove that $(\mathscr{P}(\mathscr{M}) / \sim)$ is a complete lattice, a fact which has an interesting reformulation in terms of representations.
\end{abstract}

\section{Introduction}

Let $\mathscr{M}$ be a von Neumann algebra, $\mathscr{P}(\mathscr{M})$ its projections, and $\sim$ the relation of Murray-von Neumann equivalence on $\mathscr{P}(\mathscr{M})$. The description of the quotient $(\mathscr{P}(\mathscr{M}) / \sim)$ is known as the dimension theory for $\mathscr{M}$. This is essentially the first invariant in the subject, going back to Murray and von Neumann's initial observations [24, Part II]. Among other uses, dimension theory leads directly to the type decomposition, classifies representations (see Section 7), and supports the generalized Fredholm theory [1], [2], [27] required for noncommutative geometry. In this paper we prove basic results about three aspects of dimension theory: topology, parameterization, and order.

The second section of the paper contains background which is relevant for all three topics. Section 3 deals with topology; Sections 4 and 5 with parameterization; Sections 6 and 7 with order structure. Except for one or two references, these three groupings are independent from each other. In the remainder of this introduction we explain the problems which motivate our investigations.

Topology. The first goal requires little explanation.

Problem 1.1. Study the topology that $(\mathscr{P}(\mathscr{M}) / \sim)$ inherits from the strong (equivalently, the weak) topology on $\mathscr{M}$.

Received February 1, 2006. 
Some of the results are used in the author's recent work on unitary orbits [32].

Parameterization. It is easy to check that $(\mathscr{P}(\mathscr{M}) / \sim)$ inherits a well-defined partial order from $\mathscr{P}(\mathscr{M})$. The classical work of Murray and von Neumann [24] and Dixmier [5], [7] shows that $(\mathscr{P}(\mathscr{M}) / \sim)$ can be naturally parameterized by a subset of the extended positive cone of the center, at least when $\mathcal{M}$ is $\sigma$-finite, so that $(\mathscr{P}(\mathscr{M}) / \sim)$ is represented as a set of $[0,+\infty]$-valued functions. This parameterization map, called a dimension function, can be extended to all of $\mathcal{M}_{+}$, and the extension is called an extended center-valued trace. The existence of a dimension function on a non- $\sigma$-finite von Neumann algebra is also classical, though not quite as well-known. It was originally studied in connection with spatial isomorphisms by Griffin [11], [12] and Pallu de la Barrière [29], and eventually given a representation-free foundation by Tomiyama [34].

There is a noticeable gap between the last two objects.

Problem 1.2. Is there a version of the extended center-valued trace which extends the dimension function on a non- $\sigma$-finite von Neumann algebra?

One might expect (and dread) technical constructions involving cardinals and limits. We show how to avoid most of this by simply marrying Tomiyama's dimension function to the Kadison-Pedersen equivalence relation $\approx$, which is the appropriate extension of $\sim$ from $\mathscr{P}(\mathscr{M})$ to $\mathscr{M}_{+}[19]$. (So $\left(\mathscr{M}_{+} / \approx\right)$ is the set of "sizes" of elements in $\mathscr{M}_{+}$.) In fact, the main point to settle (Proposition 4.3) does not involve cardinals.

Unfortunately, at high cardinality the dimension function is not normal. This entails that the offspring of the marriage mentioned above, which we call a fully extended center-valued trace, is not normal in general either.

Order. The range of Tomiyama's map consists of certain cardinal-valued order-continuous functions on the spectrum of the center. Tomiyama assumed pointwise order and arithmetic on the range, then gave some examples to show that his map lacks basic continuity properties. But one should not expect pointwise operations to behave well on infinite sets of functions, and it seems to us that these are essentially the wrong operations to be considering. Our viewpoint here is more algebraic. This repairs certain degeneracies and allows us to resolve affirmatively the basic

Problem 1.3. Is $(\mathscr{P}(\mathscr{M}) / \sim)$ always a complete lattice?

With minor changes, our method also applies to the "size theory" $\left(\mathscr{M}_{+} / \approx\right)$. We recall that a lattice (resp. complete lattice) is a partially-ordered set in which one may take meets and joins of finitely (resp. arbitrarily) many 
elements. Although $\mathscr{P}(\mathscr{M})$ is a complete lattice, it does not induce lattice operations on $(\mathscr{P}(\mathscr{M}) / \sim)$ : for example, $[p] \wedge[q]$ is not well-defined as $[p \wedge$ $q$ ]. Nonetheless the comparison theorem for projections readily implies that $(\mathscr{P}(\mathscr{M}) / \sim)$ is a lattice. And in a finite von Neumann algebra, the dimension function identifies $(\mathscr{P}(\mathscr{M}) / \sim)$ with a complete sublattice of $\mathscr{Z}(\mathscr{M})_{1}^{+}$. Problem 1.3 asks about the existence of meets and joins of arbitrarily large sets of equivalence classes coming from arbitrarily large von Neumann algebras.

After circulating a preprint version of this article, we were informed by Ken Goodearl that he and Franz Wehrung have also solved Problem 1.3 in a very recent memoir on dimension theory [10, Theorem 5-4.5]. Their work encompasses much more than von Neumann algebras, and it naturally requires quite a bit of abstract machinery. While we heartily recommend the memoir for its power, it still seems worthwhile to offer here a direct, two-page proof. (Also note that our results for $\left(\mathscr{M}_{+} / \approx\right)$ are not covered by [10].) In the last section of the paper we reinterpret the lattice structure in terms of representations.

\section{Background}

This section covers the classical dimension theory which is used repeatedly in the paper. Though most of this is standard material, we will arrive at reinterpretations and extensions, especially in Sections 4-6. We therefore present it in some detail.

Let $\mathcal{M}$ be a von Neumann algebra of arbitrary type and cardinality. We write $\mathscr{Z}(\mathscr{M})$ for its center, and we occasionally symbolize the strong and weak topologies by $s$ and $w$. The closure of a set in a topological space is denoted by a bar, e.g. $\bar{E}$. The central support of an operator $x$ is $c(x)$, and its spectrum is $\operatorname{sp}(x)$.

We use the standard terminology and results from [33, Section V.1] for projections, including $p^{\perp}$ for $(1-p)$. Besides $p \sim q$, we write $p \preccurlyeq q$ for subequivalence, and $p \prec q$ for $p \preccurlyeq q$ but not $p \sim q$. Notice that for pairwise orthogonal sets $\left\{p_{\alpha}\right\},\left\{q_{\alpha}\right\} \subset \mathscr{P}(\mathscr{M})$,

$$
\begin{aligned}
& p_{\alpha} \sim q_{\alpha}, \forall \alpha \Rightarrow\left(\sum p_{\alpha}\right) \sim\left(\sum q_{\alpha}\right), \\
& p_{\alpha} \preccurlyeq q_{\alpha}, \forall \alpha \Rightarrow\left(\sum p_{\alpha}\right) \preccurlyeq\left(\sum q_{\alpha}\right) .
\end{aligned}
$$

According to $(2.1)$, we can sum unambiguously any set in $(\mathscr{P}(\mathscr{M}) / \sim)$ for which there are mutually orthogonal representatives, simply by taking the equivalence class of the sum of representatives. This determines a partial order on $(\mathscr{P}(\mathscr{M}) / \sim):[p] \leq[q]$ if there exists a projection $r$ with $[p]+[r]=[q]$. It is easy to check that $\left[p_{1}\right] \leq\left[p_{2}\right]$ means nothing other than $p_{1} \preccurlyeq p_{2}$. 
Actually the comparison theorem for projections [33, Theorem V.1.8] implies that $(\mathscr{P}(\mathscr{M}) / \sim)$ is a lattice. For $p, q \in \mathscr{P}(\mathscr{M})$, let $z$ be a central projection with $z p \preccurlyeq z q, z^{\perp} p \succcurlyeq z^{\perp} q$. Then

$$
[p] \wedge[q]=\left[z p+z^{\perp} q\right], \quad[p] \vee[q]=\left[z^{\perp} p+z q\right] .
$$

Next we recall basic properties of the extended center-valued trace. This material is due to Dixmier [5], [7], but for the reader's convenience (presumably), we give citations from Takesaki's book [33].

Definition 2.1 ([33, Definition V.2.33]). Let $\mathcal{M}$ be an arbitrary von Neumann algebra, and let $\Omega(\mathscr{Z}(\mathscr{M}))$ be the spectrum of the abelian $C^{*}$-algebra $\mathscr{Z}(\mathscr{M})$. By $\widehat{\mathscr{Z}(\mathscr{M})_{+}}$we mean the partially-ordered monoid of $[0,+\infty]$-valued continuous functions on $\Omega(\mathscr{Z}(\mathscr{M}))$. The cone $\mathscr{Z}(\mathscr{M})_{+}$is contained in $\widehat{\mathscr{Z}(\mathscr{M})_{+}}$ and acts on it by multiplication.

An extended center-valued trace on $\mathscr{M}$ is an additive map $T: \mathscr{M}_{+} \rightarrow$ $\widehat{\mathscr{Z}(\mathscr{M})_{+}}$which satisfies the following: (1) $T\left(y^{*} y\right)=T\left(y y^{*}\right)$ for $y \in \mathscr{M}$; (2) $T(z x)=z T(x)$ for $z \in \mathscr{Z}(\mathscr{M})_{+}$and $x \in \mathscr{M}_{+}$.

$T$ is faithful if $T\left(x^{*} x\right)=0 \Rightarrow x=0, \forall x \in \mathscr{M}_{+} . T$ is normal if

$$
T\left(\sup x_{\alpha}\right)=\sup T\left(x_{\alpha}\right)
$$

for any bounded increasing net $\left\{x_{\alpha}\right\} \subset \mathscr{M}_{+}$. T is semifinite if the linear span of $\left\{x \in \mathscr{M}_{+} \mid T(x) \in \mathscr{Z}(\mathscr{M})_{+}\right\}$is $\sigma$-weakly dense in $\mathscr{M}$.

Here we wish to draw attention to a point which will be amplified in Sections 5 and 6 . What is the meaning of the expression "sup $T\left(x_{\alpha}\right)$ " in (2.4)? The pointwise supremum of an increasing family of $[0,+\infty]$-valued continuous functions on $\Omega(\mathscr{Z}(\mathscr{M}))$ may not be continuous, and some kind of algebraic supremum is required instead. Dixmier showed that such a supremum exists, using the fact that $\Omega(\mathscr{Z}(\mathscr{M}))$ is stonean [6]. He also mentions specifically that other methods, including a purely formal one, could reach the same goal [7, p. 25]. We suppose that our technique in Section 6 is similar to the formal approach that he had in mind.

Semifinite von Neumann algebras - those with no summand of type III - are characterized by the existence of a faithful normal semifinite extended centervalued trace [33, Theorem V.2.34]. Such a map $T$ is unique up to multiplication by an element of $\widehat{\mathscr{Z}(\mathscr{M})}$ + which takes finite values on an open dense subset of $\Omega(\mathscr{Z}(\mathscr{M}))$, so all are equally useful in calculations. A projection $p$ is finite if and only if $T(p)$ takes finite values on an open dense subset of $\Omega(\mathscr{Z}(\mathscr{M}))[33$, Proposition V.2.35]. From all this $p \preccurlyeq q \Rightarrow T(p) \leq T(q)$, and the converse holds if $p$ is finite. 
If $\mathscr{M}$ is finite, there is a unique faithful extended center-valued trace $T$ with $T\left(1_{\mathscr{M}}\right)=1_{\mathscr{M}}[33$, Theorem V.2.6]. Such a map is automatically normal, and the linear extension which is defined on all of $\mathscr{M}$ is called simply a centervalued trace.

Convention 2.2. Whenever we talk of an "extended center-valued trace" $T$ on $\mathscr{M}_{+}$in the sequel, it is assumed that

- $T$ is normal and faithful;

- on the finite summand of $\mathscr{M}, T$ agrees with the center-valued trace;

- on the semifinite summand of $\mathscr{M}, T$ is semifinite;

- on the infinite type I summand of $\mathscr{M}, T$ maps an abelian projection to its central support.

Therefore any two extended center-valued traces agree off the type $\mathrm{II}_{\infty}$ summand, and in particular $T(h)=(+\infty) c(h)$ when $h$ is supported on the type III summand. (Here $c(h)$ is identified with the characteristic function of a clopen set in $\Omega(\mathscr{Z}(\mathscr{M}))$.)

A word about operator topologies on $\mathscr{M}$ : the strong, $\sigma$-strong, weak, and $\sigma$-weak topologies can all be defined spatially. The $\sigma$-strong and $\sigma$-weak topologies are independent of the choice of (faithful normal) representation, and this is not true for the strong and the weak. But on bounded sets, we have the agreements strong $=\sigma$-strong and weak $=\sigma$-weak; we therefore permit ourselves the small linguistic abuse of referring to the strong or weak topology on a bounded subset of (unrepresented) $\mathscr{M}$.

For $\mathscr{M}$ finite, the normality of the center-valued trace is equivalent to $\sigma$-weak- $\sigma$-weak continuity. It will be more useful for us that this map is also $\sigma$-strong- $\sigma$-strong continuous, and therefore strong-strong continuous on bounded sets. (See [11, Theorem 13], [8, I.4.Théorème 2 and p. 250], and [30] in connection with this. In fact the strong-strong or weak-weak continuity on all of $\mathscr{M}$ does depend on the representation [11, Theorem 8].)

Here are some examples of $(\mathscr{P}(\mathscr{M}) / \sim)$ for factors. With $T$ an extended center-valued trace on $\mathscr{M}$, the isomorphisms (2)-(4) are effected by the map $[p] \mapsto T(p)$.

(1) When $\mathscr{M}$ is a type $\mathrm{I}_{\kappa}$ factor, $(\mathscr{P}(\mathscr{M}) / \sim)$ is isomorphic to the initial segment of cardinals $\leq \kappa$, via the map that sends a projection to its rank.

(2) When $\mathscr{M}$ is a type $\mathrm{II}_{1}$ factor, $(\mathscr{P}(\mathscr{M}) / \sim) \simeq[0,1]$.

(3) When $\mathscr{M}$ is a $\sigma$-finite type $\mathrm{II}_{\infty}$ factor, $(\mathscr{P}(\mathscr{M}) / \sim) \simeq[0,+\infty]$.

(4) When $\mathscr{M}$ is a $\sigma$-finite type III factor, $(\mathscr{P}(\mathscr{M}) / \sim) \simeq\{0,+\infty\}$.

Note that $T$ loses some of its utility on non- $\sigma$-finite factors; being capped at " $+\infty$ ", it cannot distinguish between infinite cardinals. 
But continuous (type II) and degenerate (type III) dimension theories were part of the original appeal for Murray and von Neumann: what does happen at large cardinality? Since $(\mathscr{P}(\mathscr{M}) / \sim)$ is totally ordered if and only if $\mathscr{M}$ is a factor, this is the scenario closest to set theory. Do type II and III factors contain "quantum cardinal arithmetic" which diverges from the usual cardinal arithmetic of a type I factor?

The questions above are answered neatly by the parameterization of $(\mathscr{P}(\mathscr{M}) / \sim)$ as developed by Griffin [11], [12], Pallu de la Barrière [29], and especially as formulated by Tomiyama [34]. The main point is a structure theorem allowing us to break a properly infinite von Neumann algebra into direct summands, each of which has a well-defined size. This is in direct analogy to the structure theorem for type I von Neumann algebras, but we use $\sigma$-finiteness instead of abelianness as the "unit of measurement".

Definition 2.3. ([34, Definition 1]) Let $\kappa$ be a cardinal. We say that a nonzero projection $p$ in a von Neumann algebra $\mathcal{M}$ is $\kappa$-homogeneous if $p$ is the sum of $\kappa$ mutually equivalent projections, each of which is the sum of centrally orthogonal $\sigma$-finite projections. We also define

$$
\kappa_{\mathscr{M}}=\sup \{\kappa \mid \mathcal{M} \text { contains a } \kappa \text {-homogeneous projection }\} .
$$

Remark 2.4. The terminology here is conflicting. We follow Tomiyama, but elsewhere " $\kappa$-homogeneous projection" can mean a central projection which is the sum of $\kappa$ equivalent abelian projections (e.g. [33, p. 299]).

A projection can be $\kappa$-homogeneous for at most one $\kappa \geq \aleph_{0}$; also for $\kappa \geq \aleph_{0}$, two $\kappa$-homogeneous projections with identical central support are necessarily equivalent [12], [34]. Note that $\kappa_{\mathcal{M}}$ is not larger than the dimension of a Hilbert space on which $\mathscr{M}$ is faithfully represented.

The fundamental result for us is a mélange of two theorems of Griffin, one covering the semifinite case (slightly adapted to our setting, and also proved by Pallu de la Barrière) and one covering the purely infinite. It was rewritten in the non-spatial setting by Tomiyama.

Theorem 2.5 ([11, Theorem 3], [12, Theorem 1], [29, Théorème I.V.1], [34, Theorem 1]). Let $\mathscr{M}$ be a properly infinite von Neumann algebra. Then uniquely

$$
1_{M}=\sum_{\aleph_{0} \leq \kappa \leq \kappa_{M}} z_{\kappa}
$$

where each $z_{\kappa}$ is either zero or a $\kappa$-homogeneous central projection.

Let $T$ be an extended center-valued trace on a von Neumann algebra $\mathscr{M}$ (following Convention 2.2). Given any $p \in \mathscr{P}(\mathscr{M})$, let $z^{f}$ be the largest central 
projection such that $z^{f} p$ is finite. By applying Theorem 2.5 to $\left(1-z^{f}\right) p \mathscr{M} p$, there are unique central projections $\left(z_{\kappa}\right)_{\aleph_{0} \leq \kappa \leq \kappa_{\mathcal{M}}}$ such that $\sum z_{\kappa} p=\left(1-z^{f}\right) p$ and any nonzero $z_{\kappa} p$ is $\kappa$-homogeneous. Make the formal assignment

$$
p=\left(z^{f} p+\sum_{\aleph_{0} \leq \kappa \leq \kappa_{\mathcal{M}}} z_{\kappa} p\right) \mapsto\left(T\left(z^{f} p\right)+\sum_{\aleph_{0} \leq \kappa \leq \kappa_{\mathcal{M}}} \kappa z_{\kappa}\right) .
$$

From our earlier comments this assignment is a complete invariant for the equivalence class of $p$.

We now describe how the right-hand side of (2.5) determines an (order) continuous function on $\Omega\left(\mathscr{Z}(\mathscr{M})\right.$ ). Identify each term $\kappa z_{\kappa}$ with the constant function $\kappa$ defined only on the clopen set corresponding to $z_{\kappa}$. Similarly realize $T\left(z^{f} p\right)$ as a continuous function on the clopen set for $z^{f}$. Their sum is then a $\left([0,+\infty) \cup\left\{\kappa \mid \aleph_{0} \leq \kappa \leq \kappa_{\mathscr{M}}\right\}\right)$-valued function, continuous on an open dense domain in $\Omega(\mathscr{Z}(\mathscr{M}))$. Tomiyama showed [34, Lemma 5] that such a function extends uniquely and continuously to all of $\Omega(\mathscr{Z}(\mathscr{M}))$.

Definition 2.6 ([34]). The assignment described above, from $\mathscr{P}(\mathscr{M})$ to the continuous $\left([0,+\infty) \cup\left\{\kappa \mid \aleph_{0} \leq \kappa \leq \kappa_{\mathcal{M}}\right\}\right)$-valued functions on $\Omega(\mathscr{Z}(\mathscr{M}))$, is a (generalized) dimension function of $\mathscr{M}$.

Theorem 2.7 ([34]). Let $D$ be a dimension function of $\mathcal{M}$. Then $D$ is additive on pairs of orthogonal projections, provided that one incorporates the positive reals into cardinal arithmetic in the obvious way. We have

$$
p \preccurlyeq q \Longleftrightarrow D(p) \leq D(q), \quad \forall p, q \in \mathscr{P}(\mathscr{M}),
$$

where we use the pointwise ordering of functions on the right-hand side.

It follows that $D$ factors as

$$
\mathscr{P}(\mathscr{M}) \rightarrow(\mathscr{P}(\mathscr{M}) / \sim) \stackrel{\sim}{\rightarrow} D(\mathscr{P}(\mathscr{M})) .
$$

Here the second map is an embedding in a function space, preserving sums (when they exist) and intertwining the multiplicative $\mathscr{P}(\mathscr{Z}(\mathscr{M})$ )-action.

Corollary 2.8 .

(1) In a factor of type $\mathrm{II}_{\infty}$, the totally ordered set $(\mathscr{P}(\mathscr{M}) / \sim)$ is isomorphic to

$$
[0,+\infty) \cup\left\{\kappa \mid \aleph_{0} \leq \kappa \leq \kappa_{\mathcal{M}}\right\} .
$$

(2) In a factor of type III, the totally ordered set $(\mathscr{P}(\mathscr{M}) / \sim)$ is isomorphic to

$$
\{0\} \cup\left\{\kappa \mid \aleph_{0} \leq \kappa \leq \kappa \mathcal{M}\right\} .
$$


So any interest in "quantum cardinal arithmetic" wanes here: infinite quantum cardinals are (isomorphically) just cardinals. For axiomatic treatments of $(\mathscr{P}(\mathscr{M}) / \sim)$ and more general algebraic structures obtained as quotients of lattices, we refer the interested reader to [22], [23], [9], [10].

\section{The topology of $(\mathscr{P}(\mathscr{M}) / \sim)$}

If we want $(\mathscr{P}(\mathscr{M}) / \sim)$ to inherit a topology from $\mathscr{P}(\mathcal{M})$, there really are not so many interesting choices. The quotient of the norm topology is the discrete topology, since $\|p-q\|<1$ implies that $p$ and $q$ are unitarily equivalent [35, Proposition 5.2.6]. And all of the "operator" topologies (notably, the strong and the weak) are equivalent when restricted to $\mathscr{P}(\mathscr{M})$ [20, Exercise 5.7.4]. We point out, however, that $(\mathscr{P}(\mathscr{M})$, strong) is complete as a topological subspace of $\mathscr{M}$, while $(\mathscr{P}(\mathscr{M})$, weak) may not be; completeness is not a topological property.

We will denote by " $Q O T$ " the resulting quotient strong/weak operator topology on $(\mathscr{P}(\mathscr{M}) / \sim)$. In the rest of this section, all closures and convergences in $(\mathscr{P}(\mathscr{M}) / \sim)$ are to be understood in this topology.

LEMMA 3.1. Let $\left\{x_{\alpha}\right\}$ be a net in a von Neumann algebra $M$ equipped with an extended center-valued trace $T$. If $x_{\alpha}^{*} x_{\alpha}=y_{1}$ is fixed, while $x_{\alpha} x_{\alpha}^{*} \stackrel{w}{\rightarrow} y_{2}$, then $T\left(y_{1}\right) \geq T\left(y_{2}\right)$ in $\widehat{\mathscr{Z}(\mathscr{M})_{+}}$.

Proof. Fix any $\varphi \in \mathscr{Z}(\mathcal{M})_{*}^{+}$. Then $\varphi \circ T$ is a normal weight, so weakly lower-semicontinuous [13]. We have

$$
\begin{aligned}
\varphi \circ T\left(y_{2}\right) & =\varphi \circ T\left(w-\lim x_{\alpha} x_{\alpha}^{*}\right) \leq \liminf \varphi \circ T\left(x_{\alpha} x_{\alpha}^{*}\right) \\
& =\liminf \varphi \circ T\left(x_{\alpha}^{*} x_{\alpha}\right)=\varphi \circ T\left(y_{1}\right) .
\end{aligned}
$$

Since $\varphi$ is arbitrary, the conclusion follows.

Lemma 3.2. Let $\mathcal{M}$ be a von Neumann algebra.

(1) Let $\left\{p_{j}\right\}_{j=1}^{n}$ be equivalent properly infinite projections in $\mathcal{M}$, where $n \in$ $(\mathrm{N} \cup\{\infty\})$. Then $p_{1} \sim \vee p_{j}$.

(2) Let $p \preccurlyeq q$ be properly infinite projections in $\mathcal{M}$ with equal central support. Then the set

$$
\left\{p_{\alpha} \mid p \sim p_{\alpha} \leq q\right\}
$$

equipped with the usual operator ordering, is a net which converges strongly to $q$.

Proof. (1) It is clear that $p_{1} \preccurlyeq \vee p_{j}$. Write $p_{1}=\sum q_{j}$, where each $q_{j} \sim p_{1}$. Let $\left\{v_{j}\right\}$ be partial isometries with $v_{j}^{*} v_{j}=p_{j}, v_{j} v_{j}^{*}=q_{j}$. Since the $q_{j}$ are 
orthogonal, we have

$$
v_{i}^{*} v_{j}=v_{i}^{*} v_{i} v_{i}^{*} v_{j} v_{j}^{*} v_{j}=v_{i}^{*} q_{i} q_{j} v_{j}=0, \quad i \neq j .
$$

Let $x$ be the bounded operator $\sum\left(v_{j} / 2^{j}\right)$. Using (3.2),

$$
x^{*} x=\sum\left(p_{j} / 4^{j}\right),
$$

so that the right support of $x$ is $\vee p_{j}$. (By definition, the right (resp. left) support of $x$ is the smallest projection $p$ such that $x=x p$ (resp. $x=p x$ ).) From its form as a sum, the left support of $x$ is less than or equal to the join of the left supports of $v_{j}$, which is $\vee q_{j}=p_{1}$. Since the left and right supports of an operator are always equivalent [33, Proposition V.1.5], we have $p_{1} \succcurlyeq \vee p_{j}$ as well.

(2) By renaming $q \mathscr{M} q$ as $\mathscr{M}$ and replacing $p$ with an equivalent projection under $q$, it suffices to prove the statement for $q=1$. First note that (3.1) is upward directed, by part (1) applied to two projections. Therefore it is a bounded increasing net which converges strongly to its supremum $r$. We claim $r=1$. To see this, use the comparison theorem on $p$ and $r^{\perp}$ to find $s \in \mathscr{P}(\mathscr{M})$ and $z \in \mathscr{P}(\mathscr{Z}(\mathscr{M}))$ satisfying

$$
s \sim p, \quad s z \geq r^{\perp} z, \quad s z^{\perp} \leq r^{\perp} z^{\perp} .
$$

From its definition as a supremum, $r \geq s$, so from (3.3) we deduce

$$
s z^{\perp}=0, \quad r^{\perp} z=0 .
$$

As $c(s)=c(p)=1$, the first equation of (3.4) implies $z^{\perp}=0$, and the second then gives $r^{\perp}=0$.

Theorem 3.3 ([21, Theorem 8.4.4]). Let $\mathcal{M}$ be finite with center-valued trace T. If $\mathscr{M}$ is type $\mathrm{I}_{n}, T(\mathscr{P}(\mathscr{M}))=\left\{z \in \mathscr{Z}(\mathscr{M})_{1}^{+} \mid \operatorname{sp}(z) \subseteq\left\{0, \frac{1}{n}, \frac{2}{n}, \ldots, 1\right\}\right\}$. If $\mathscr{M}$ is type $\mathrm{II}_{1}, T(\mathscr{P}(\mathscr{M}))=\mathscr{Z}(\mathscr{M})_{1}^{+}$.

For use in Section 6, we remark that the proof of Theorem 3.3 (see [21, Theorem 8.4.4]) also gives the following equalities. If $\mathscr{M}$ is infinite type I, $T(\mathscr{P}(\mathscr{M}))$ consists of the functions in $\widehat{\mathscr{Z}(\mathscr{M})_{+}}$with range in $\{0,1,2, \ldots,+\infty\}$, and $T\left(\mathscr{M}_{1}^{+}\right)=\widehat{\mathscr{Z}(\mathscr{M})_{+}}$. If $\mathscr{M}$ is type $\mathrm{II}_{\infty}, T(\mathscr{P}(\mathscr{M}))=\widehat{\mathscr{Z}(\mathscr{M})_{+}}$.

We now identify $Q O T$, keeping in mind that the strong and weak topologies on $\mathscr{Z}(\mathscr{M})_{1}^{+}$are typically not equivalent.

THEOREM 3.4. If $\mathscr{M}$ is a finite von Neumann algebra with center-valued trace $T$, the map $[p] \mapsto T(p)$ implements a homeomorphism from 
$((\mathscr{P}(\mathscr{M}) / \sim), Q O T)$ to a subspace of $\left(\mathscr{Z}(\mathscr{M})_{1}^{+}\right.$, strong). Consequently

$$
\overline{\{[p]\}}=\{[p]\}, \quad p \in \mathscr{P}(\mathscr{M}) .
$$

Proof. If $\left[p_{\alpha}\right] \rightarrow[p]$, then there exist $q_{\alpha} \sim p_{\alpha}$ with $q_{\alpha} \stackrel{s}{\rightarrow} p$. By the strong-strong continuity of $T$ on bounded sets, we have $T\left(p_{\alpha}\right)=T\left(q_{\alpha}\right) \stackrel{s}{\rightarrow}$ $T(p)$.

On the other hand, suppose $p_{\alpha}, p$ are projections such that $T\left(p_{\alpha}\right) \stackrel{s}{\rightarrow} T(p)$. Using the comparison theorem again, let $\left\{q_{\alpha}\right\}$ and $\left\{z_{\alpha}\right\}$ be sets of projections such that

$$
q_{\alpha} \sim p_{\alpha}, \quad z_{\alpha} \in \mathscr{Z}(\mathscr{M}), \quad q_{\alpha} z_{\alpha} \geq p z_{\alpha}, \quad q_{\alpha} z_{\alpha}^{\perp} \leq p z_{\alpha}^{\perp} .
$$

When $\mathscr{M}$ is $\sigma$-finite, the strong topology on bounded sets is generated by the norm $x \mapsto \varphi\left(x^{*} x\right)^{1 / 2}$, for $\varphi$ any faithful normal state [33, Proposition III.5.3]. A general finite algebra is a direct sum of $\sigma$-finite ones [33, Corollary V.2.9], so it suffices to show convergence for the seminorms coming from a family of normal tracial states, each of which is faithful on a $\sigma$-finite summand.

We now take such a trace $\tau$ and compute

$$
\begin{aligned}
\tau\left(\left|q_{\alpha}-p\right|^{2}\right) & =\tau\left(\left(q_{\alpha}-p\right) z_{\alpha}+\left(p-q_{\alpha}\right) z_{\alpha}^{\perp}\right) \\
& =\tau\left(\left(T\left(q_{\alpha}\right)-T(p)\right) z_{\alpha}+\left(T(p)-T\left(q_{\alpha}\right)\right) z_{\alpha}^{\perp}\right) \\
& =\tau\left(\left|T\left(q_{\alpha}\right)-T(p)\right|\right) \\
& =\tau\left(\left|T\left(p_{\alpha}\right)-T(p)\right|\right) \\
& \leq \tau\left(\left|T\left(p_{\alpha}\right)-T(p)\right|^{2}\right)^{1 / 2} \rightarrow 0 .
\end{aligned}
$$

The last step uses the noncommutative Hölder inequality (cf. [26, Eq. 24]).

THEOREM 3.5. Let $p$ be a projection in a properly infinite von Neumann algebra M. If $p$ is finite,

$$
\overline{\{[p]\}}=\{[q] \mid[q] \leq[p]\} .
$$

If $p$ is properly infinite and $c(p)=1_{\mathscr{M}}$,

$$
\overline{\{[p]\}}=(\mathscr{P}(\mathscr{M}) / \sim) .
$$

Equations (3.5) and (3.6) may be synthesized into

$$
\overline{\{[p]\}}=\{[q] \mid T(q) \leq T(p)\}, \quad \forall p \in \mathscr{P}(\mathscr{M}),
$$

for any extended center-valued trace $T$. 
Proof. We prove the theorem by showing three separate implications, one per paragraph.

Let $p$ be finite, and suppose that $[q] \in \overline{\{[p]\}}$, so that there are $p_{\alpha} \sim p$ with $p_{\alpha} \stackrel{w}{\rightarrow} q$. With $T$ an extended center-valued trace, Lemma 3.1 gives $T(q) \leq T(p)$. We have assumed $p$ finite, so $q$ is as well, and both are supported on the semifinite summand of $\mathcal{M}$. We may conclude $[q] \leq[p]$.

Now let $p$ be properly infinite with $c(p)=1$, and suppose that $[q] \geq[p]$. By Lemma 3.2(2), $p_{\alpha} \stackrel{s}{\rightarrow} q$, where $\left\{p_{\alpha}\right\}$ is the net in (3.1). This shows $[q] \in$ $\overline{\{[p]\}}$.

By dividing $\mathcal{M}$ up into central summands, all of (3.5), (3.6), and (3.7) will now be settled if we show that $[q] \leq[p]$ entails $[q] \in \overline{\{[p]\}}$ (with no assumptions on $p$ and $q$ ). Replacing $q$ by an equivalent projection if necessary, we may assume that $q^{\perp} \sim 1$. (Write $1=\left(s_{1}+s_{2}\right) \sim s_{1} \sim s_{2}$, and find a projection less than $s_{1}$ which is equivalent to $q$.) Let $q \sim p_{0} \leq p$. Also write $q^{\perp}=\sum_{j=1}^{\infty} q_{j}$, with $q_{j} \sim 1$. Finally find $\left\{r_{j}\right\}$ with $\left(p-p_{0}\right) \sim r_{j} \leq q_{j}$. Now

$$
p=\left(p_{0}+\left(p-p_{0}\right)\right) \sim\left(q+r_{j}\right) \stackrel{s}{\rightarrow} q
$$

as required.

Corollary 3.6. Let $M$ be a factor, $T$ an extended center-valued trace on $\mathcal{M}$, and $E \subseteq(\mathscr{P}(\mathscr{M}) / \sim)$. If $\mathcal{M}$ is finite,

$$
\bar{E}=\{[q] \mid T(q) \in \overline{\{T(p) \mid[p] \in E\}}\},
$$

where $T(\mathscr{P}(\mathscr{M}))$ has its usual topology as a subset of $[0,1]$. If $\mathscr{M}$ is properly infinite,

$$
\bar{E}=\left\{[q] \mid T(q) \leq \sup _{[p] \in E} T(p)\right\} .
$$

Corollary 3.7. QOT is a $T_{1}$ topology exactly when $\mathscr{M}$ is finite.

Proof. A topology is $T_{1}$ if and only if for any two distinct points $x, y$, there is a closed set which contains $x$ and not $y$. Equivalently, a topology is $T_{1}$ if and only if singletons are closed.

Theorem 3.4 explicitly says that $Q O T$ is $T_{1}$ in finite algebras. If $\mathscr{M}$ contains a properly infinite projection $p,[0] \in \overline{\{[p]\}}$ by Theorem 3.5 .

It turns out to be more useful for our applications elsewhere [32, Theorem 5.4] to know when $Q O T$ is $T_{0}$. A topology is $T_{0}$ if for any two distinct points, there exists a closed set which contains exactly one of them.

Proposition 3.8. For a von Neumann algebra $\mathcal{M}$, the following conditions are equivalent. 
(1) QOT is a $T_{0}$ topology on $(\mathscr{P}(\mathscr{M}) / \sim)$.

(2) For any $p, q \in \mathscr{P}(\mathscr{M}),[p] \in \overline{\{[q]\}} \Rightarrow p \preccurlyeq q$.

(3) $\kappa_{\mathcal{M}} \leq \aleph_{0}$.

(4) $\mathcal{M}$ does not contain $\mathscr{B}\left(\mathfrak{S}_{1}\right)$, where $\mathfrak{S}_{1}$ is a Hilbert space of dimension $\aleph_{1}$.

(5) $\mathcal{M}$ is a (possibly uncountable) direct sum of $\sigma$-finite von Neumann algebras.

Proof. The equivalence of conditions (3)-(5) follows from the definitions and Theorem 2.5. We therefore focus on the equivalence of (1)-(3).

(1) $\rightarrow$ (3): If (3) fails, let $q$ be an $\aleph_{1}$-homogeneous projection, and let $p$ be an $\aleph_{0}$-homogeneous projection with $c(p)=c(q)$. Then $[p] \in \overline{\{[q]\}}$ and $[q] \in \overline{\{[p]\}}$, but $[p] \neq[q]$. Clearly there is no closed set separating the two.

(3) $\rightarrow(2)$ : When $\kappa_{\mathscr{M}} \leq \aleph_{0},\left.T\right|_{\mathscr{P}(\mathcal{M})}$ can be identified with $D$. By Theorems 3.4 and 3.5 we have

$$
[p] \in \overline{\{[q]\}} \Rightarrow T(p) \leq T(q) \Rightarrow D(p) \leq D(q) \Rightarrow p \preccurlyeq q .
$$

$(2) \rightarrow(1)$ : Suppose (2) holds, and let $[p],[q] \in(\mathscr{P}(\mathscr{M}) / \sim)$ be such that they cannot be separated by a closed set. Then

$$
[p] \in \overline{\{[q]\}},[q] \in \overline{\{[p]\}} \Rightarrow p \preccurlyeq q, q \preccurlyeq p \Rightarrow[p]=[q] .
$$

\section{From dimension function to trace in full generality}

Let $T$ be an extended center-valued trace on a von Neumann algebra $\mathscr{M}$, with $D$ the induced dimension function. We will create a map which extends $D$ to the entire positive cone and so is a trace which distinguishes among infinite cardinalities. (In case $\kappa_{\mathscr{M}} \leq \aleph_{0}$, this process simply recovers $T$.) The main tool is Kadison-Pedersen equivalence.

Definition 4.1 ([19]). For two elements $h, k \in \mathcal{M}_{+}$, we write $h \approx k$ if and only if there exists a family $\left\{x_{\alpha}\right\} \subset \mathscr{M}$ such that $h=\sum x_{\alpha}^{*} x_{\alpha}$ and $k=\sum x_{\alpha} x_{\alpha}^{*}$.

We write $h \lesssim k$ to mean that there exists $k^{\prime} \leq k$ with $h \approx k^{\prime}$.

For $h \in \mathcal{M}_{+}$, we say that $h$ is finite if $h \approx k \leq h \Rightarrow k=h$.

The following facts are shown in [19].

- The relation $\approx$ is an equivalence relation. It is homogeneous $(h \approx k \Rightarrow$ $\lambda h \approx \lambda k, \lambda \in \mathrm{R}_{+}$) and completely additive in the sense that

$$
h_{\alpha} \approx k_{\alpha}, \forall \alpha \Rightarrow \sum h_{\alpha} \approx \sum k_{\alpha}
$$

(when the two sums exist in $\mathscr{M}$ ). 
- The relation $\lesssim$ gives a partial order on equivalence classes. In particular,

$$
h \lesssim k, k \lesssim h \Rightarrow h \approx k, \quad h, k \in \mathscr{M}_{+} .
$$

- For projections, $p \approx q \Longleftrightarrow p \sim q$.

- For $h, k \in \mathcal{M}_{+}, h \lesssim k \Rightarrow T(h) \leq T(k)$, and the converse holds if $h$ is finite.

We will also say that nonzero $h \in \mathscr{M}_{+}$is properly infinite if $z h$ is finite and nonzero for no central projection $z$. For projections, the usage here of "finite" and "properly infinite" coincides with the usual meaning; in fact proper infiniteness of (nonzero) $h$ in either case is characterized by $T(h)$ being $\{0,+\infty\}$ valued.

LeMma 4.2.

(1) Let $\lambda \in(0,1) \cup(1, \infty)$, and let $p$ be a projection. Then

$$
p \text { is properly infinite } \Longleftrightarrow p \approx \lambda p \text {. }
$$

(2) Let $h, k \in \mathcal{M}_{+}$have equal central support, with $k$ properly infinite and $h$ a countable sum of finite elements. Then $h \lesssim k$.

(3) Let $h, k \in M_{+}$be properly infinite with equal central support, and suppose that each is a countably infinite sum of finite elements. Then $h \approx k$.

Proof. (1) If $p \approx \lambda p$, then $T(p)$ must be $\{0,+\infty\}$-valued. For the opposite implication, we first check rational multiples. Let $m, n \in \mathrm{N}$. By proper infiniteness, we may write

$$
p=\sum_{i=1}^{m} p_{i}=\sum_{j=1}^{n} p_{j}^{\prime}, \quad p_{i} \sim p \sim p_{j}^{\prime}, \forall i, j .
$$

Then

$$
\begin{aligned}
p & =\sum_{i=1}^{m} p_{i} \approx \sum_{i=1}^{m} p=m p=\left(\frac{m}{n}\right) n p=\left(\frac{m}{n}\right)\left(\sum_{j=1}^{n} p\right) \\
& \approx\left(\frac{m}{n}\right)\left(\sum_{j=1}^{n} p_{j}^{\prime}\right)=\left(\frac{m}{n}\right) p .
\end{aligned}
$$

Find two positive rationals $\lambda_{1}, \lambda_{2}$ with $\lambda_{1} \leq \lambda \leq \lambda_{2}$ :

$$
p \approx \lambda_{1} p \leq \lambda p \leq \lambda_{2} p \approx p \Rightarrow p \approx \lambda p
$$

using (4.1). 
(2) Write $h=\sum_{j=1}^{\infty} h_{j}$, where each $h_{j}$ is finite. Since $T\left(h_{1}\right) \leq T(k)$, there is an operator $k_{1}$ with $h_{1} \approx k_{1} \leq k$. We continue in this way: since $T\left(h_{n}\right) \leq T\left(k-\sum_{j=1}^{n-1} k_{j}\right)$, find $k_{n}$ with $h_{n} \approx k_{n} \leq\left(h-\sum_{j=1}^{n-1} k_{j}\right)$.

Now each $\left(\sum_{j=1}^{n} h_{j}\right) \approx\left(\sum_{j=1}^{n} k_{j}\right)$, and these terms are finite and increasing to $h$ and some $k^{\prime}$, respectively. It follows from [19, Lemma 3.3] that $h \approx k^{\prime} \leq k$.

(3) Both $h \lesssim k$ and $h \gtrsim k$ follow from the previous part; apply (4.1).

Proposition 4.3. Let $h \in M_{+}$be properly infinite. Then there exists $p \in$ $\mathscr{P}(\mathscr{M})$ such that $h \approx p$.

PRoOF. It does no harm to assume that $h$ has full central support, and therefore $\mathcal{M}$ is properly infinite. Write the identity as $1_{\mathcal{M}}=\sum_{n=-\infty}^{\infty} p_{n}, 1_{\mathcal{M}} \sim$ $p_{n}$, and let $r_{0} \leq p_{0}$ be an $\aleph_{0}$-homogeneous projection with full central support.

Now make the decomposition

$$
h=\sum_{n=1}^{\infty}\left(2^{-n}\|h\|\right) q_{n},
$$

where $q_{n}$ is the spectral projection for $h$ corresponding to

$$
\bigcup_{j=1}^{2^{n-1}}\left((2 j-1) 2^{-n}\|h\|,(2 j) 2^{-n}\|h\|\right] .
$$

For each $n \geq 1$, let $z_{n}^{f}$ be the largest central projection such that $z_{n}^{f} q_{n}$ is finite. Using Lemma 4.2(1) and then conjugating by a partial isometry from $\left(1-z_{n}^{f}\right)$ to $\left(1-z_{n}^{f}\right) p_{n}$, find a projection $r_{n}$ with

$$
\left(1-z_{n}^{f}\right)\left(2^{-n}\|h\|\right) q_{n} \approx\left(1-z_{n}^{f}\right) q_{n} \sim r_{n} \leq p_{n} .
$$

Conjugating by a partial isometry from $z_{n}^{f}$ to $z_{n}^{f} p_{-n}$, let $r_{-n}$ be any operator (necessarily finite, but not necessarily a projection) with

$$
z_{n}^{f}\left(2^{-n}\|h\|\right) q_{n} \approx r_{-n} \in p_{-n} M p_{-n} .
$$

By construction we have $h \approx \sum_{n=1}^{\infty}\left(r_{n}+r_{-n}\right)$.

Set $z_{0}=\wedge z_{n}^{f}$. We will complete the proof by showing that $z_{0} h$ and $z_{0}^{\perp} h$ are both (Kadison-Pedersen) equivalent to projections.

First,

$$
z_{0} h \approx z_{0}\left(\sum_{n=1}^{\infty}\left(r_{n}+r_{-n}\right)\right)=z_{0}\left(\sum_{n=1}^{\infty} r_{-n}\right) .
$$


The left-hand side has central support $z_{0}$, and is either zero or properly infinite because $h$ is properly infinite. The right-hand side is a countable sum of finite elements. By Lemma 4.2(3),

$$
z_{0} h \approx z_{0} r_{0}
$$

Second,

$$
z_{0}^{\perp}\left(\sum_{n=1}^{\infty} r_{n}\right) \sim z_{0}^{\perp}\left(r_{0}+\sum_{n=1}^{\infty} r_{n}\right),
$$

since the central supports are equal and the left-hand side is a properly infinite projection. (For example, this follows by evaluating the dimension function on both sides and noting that adding $\aleph_{0}$ does not change an infinite cardinal.) On the other hand, Lemma 4.2(2) implies

$$
z_{0}^{\perp}\left(\sum_{n=1}^{\infty} r_{-n}\right) \lesssim z_{0}^{\perp} r_{0} .
$$

We put these together:

$$
\begin{aligned}
z_{0}^{\perp} h & \approx z_{0}^{\perp}\left(\sum_{n=1}^{\infty}\left(r_{n}+r_{-n}\right)\right) \lesssim z_{0}^{\perp}\left(r_{0}+\sum_{n=1}^{\infty} r_{n}\right) \\
& \sim z_{0}^{\perp}\left(\sum_{n=1}^{\infty} r_{n}\right) \lesssim z_{0}^{\perp}\left(\sum_{n=1}^{\infty}\left(r_{n}+r_{-n}\right)\right) \approx z_{0}^{\perp} h
\end{aligned}
$$

Then all terms above are (Kadison-Pedersen) equivalent, and the middle two are projections.

Corollary 4.4. Under the same hypotheses as in Lemma 4.2(2), $k \approx \lambda k$ for any $\lambda \in(0, \infty)$, and $(h+k) \approx k$.

Proof. By Proposition 4.3 and Lemma 4.2(1), there is a properly infinite projection $p$ with $k \approx p \approx \lambda p \approx \lambda k$. By Lemma 4.2(2),

$$
(h+k) \lesssim 2 k \approx k \lesssim(h+k) \Rightarrow(h+k) \approx k .
$$

We are now ready to define our map.

Definition 4.5. With $T$ (and $D$ ) given, we construct a fully extended center-valued trace $\widehat{T}$ on $\mathcal{M}$ as follows.

For any $h \in \mathcal{M}_{+}$, let $z^{f}$ be the largest central projection so that $z^{f} h$ is finite. Let $p$ be a projection with $p \approx\left(1-z^{f}\right) h$. Such a $p$ exists by Proposition 4.3, and all choices belong to the same Murray-von Neumann equivalence class. 
We define

$$
\widehat{T}(h)=T\left(z^{f} h\right)+D\left(\left(1-z^{f}\right) p\right),
$$

which we view as a continuous $\left([0,+\infty) \cup\left\{\kappa \mid \aleph_{0} \leq \kappa \leq \kappa, \mu\right\}\right)$-valued function on $\Omega(\mathscr{Z}(\mathscr{M}))$.

THEOREM 4.6. The map $\widehat{T}$ extends $D$, is additive, intertwines the multiplicative action of $\mathscr{Z}(\mathscr{M})_{+}$, and satisfies

$$
h \lesssim k \Longleftrightarrow \widehat{T}(h) \leq \widehat{T}(k), \quad h, k \in \mathcal{M}_{+} .
$$

(We are allowing cardinal arithmetic to incorporate the positive reals in the obvious way.)

Proof. Clearly $\widehat{T}$ extends $D$. By the properties of $D$ and $T$ we have $h \approx$ $k \Longleftrightarrow \widehat{T}(h)=\widehat{T}(k)$.

In saying that $\widehat{T}$ is additive, we mean that

$$
\widehat{T}(h+k)=\widehat{T}(h)+\widehat{T}(k), \quad h, k \in \mathcal{M}_{+} .
$$

For $h, k$ finite, (4.4) follows from additivity of $T$. For $h, k$ properly infinite, the projection representing $h+k$ may be constructed as the sum of orthogonal representing projections for $h$ and $k ;(4.4)$ then follows from the additivity of $D$. Finally, let $h$ and $k$ have the same central support, with $h$ finite and $k$ properly infinite. In this case $\widehat{T}(h)$ is bounded above by $\aleph_{0}$, while $\widehat{T}(k) \geq \aleph_{0}$ where it is nonzero. So $\widehat{T}(h)+\widehat{T}(k)=\widehat{T}(k)$. Since $(h+k) \approx k$ by Corollary 4.4 , $\widehat{T}(h+k)=\widehat{T}(k)$ as well.

In saying that $\widehat{T}$ intertwines the action of $\mathscr{Z}(\mathscr{M})_{+}$, we mean

$$
y \widehat{T}(h)=\widehat{T}(y h), \quad y \in \mathscr{Z}(\mathscr{M})_{+}, \quad h \in \mathcal{M}_{+} .
$$

Clearly (4.5) holds for finite $h$, since the analogous formula is true for $T$. It therefore suffices to prove (4.5) under the assumption that $h$ and $y$ have full central support, with $h$ properly infinite. In this case $y \widehat{T}(h)=\widehat{T}(h)$, so we are left to show that $y h \approx h$. If $y \geq \lambda c(y)$ for some $\lambda>0$, then by Corollary 4.4

$$
h \approx \lambda h \leq y h \leq\|y\| h \approx h \Rightarrow h \approx y h .
$$

The general conclusion follows by writing $y$ as a central sum of operators which are invertible on their supports.

As for (4.3), the forward implication is a consequence of additivity. For the reverse implication, we look at central summands: where $h$ is finite, this is a property of $T$; where $h$ and $k$ are both infinite, this is a property of $D$. 
From Theorem 4.6, we see that $\widehat{T}$ factors as

$$
\mathscr{M}_{+} \rightarrow\left(\mathscr{M}_{+} / \approx\right) \stackrel{\sim}{\rightarrow} \widehat{T}\left(\mathscr{M}_{+}\right) .
$$

Here the second map is an embedding in a function space, preserving order, sums, and the multiplicative $\mathscr{Z}(\mathscr{M})_{+}$-action.

More generally, we may say that an arbitrary completely additive map on $\mathscr{M}_{+}$is tracial if and only if it factors through the quotient $\mathscr{M}_{+} \rightarrow\left(\mathscr{M}_{+} / \approx\right)$. Numerical (completely additive) traces result when the range is $[0,+\infty]$; they are " $\aleph_{0}$-truncated one-dimensional representations" of $\left(\mathscr{M}_{+} / \approx\right)$.

Remark 4.7. Kadison and Pedersen observed that all extended centervalued traces on semifinite algebras can be generated in the following manner [19, Theorem 3.8]. Fix a finite projection $p$ with full central support such that $p$ is the identity on the finite summand and is abelian on the infinite type I summand (to match Convention 2.2). Then for finite $h \in \mathcal{M}_{+}, T(h)$ is the unique element of the extended center with $h \approx T(h) p$. Already this requires a small extension of $\approx$ to unbounded sums.

With a further extension involving cardinals, $\widehat{T}$ can also be defined in this way. For general $\mathscr{M}$, let $p$ be the identity on the finite summand, abelian on the infinite type I summand, finite on the type II summand, and $\aleph_{0}$-homogeneous on the type III summand; of course $p$ should have full central support. For $h \in \mathcal{M}_{+}$, one can define $\widehat{T}(h)$ as the unique formal sum (as in (2.5)) such that $h \approx \widehat{T}(h) p$ and $\widehat{T}(h)$ takes no finite nonzero values on the type III summand. Probably this is more interesting to mention than to carry out, so we omit the details.

\section{Continuity}

In the remaininder of the paper we assume that compatible $T, D$, and $\widehat{T}$ are given on $\mathscr{M}$.

The order-preserving embeddings of $(\mathscr{P}(\mathscr{M}) / \sim)$ and $\left(\mathscr{M}_{+} / \approx\right)$ in a function space (albeit cardinal-valued) make pointwise operations available. From Theorems 2.7 and 4.6 we know that for finite sets, addition in the quotient structures agrees with addition of functions. One may likewise add up infinite sets of functions, but there is no guarantee that the sum will be continuous. Tomiyama gave an example [34, Example 2] to show that for a pairwise orthogonal set $\left\{p_{\alpha}\right\}$, one cannot expect an identity between $\sum D\left(p_{\alpha}\right)$ and $D\left(\sum p_{\alpha}\right)$, so that $D$ is not completely additive.

This is really an artifact of the function representation. There is a natural (partially-defined) sum operation on $(\mathscr{P}(\mathscr{M}) / \sim$ ), given by

$$
\sum\left[p_{\alpha}\right] \triangleq\left[\sum q_{\alpha}\right]
$$


whenever there exists a set of pairwise orthogonal projections $\left\{q_{\alpha}\right\}$ with $q_{\alpha} \sim$ $p_{\alpha}$. A similar definition is possible for sums in $\widehat{T}\left(\mathscr{M}_{+}\right)$, where we simply require that the representatives sum to an element of $\mathcal{M}_{+}$. Note that there is no ambiguity in these definitions, by (2.1) and the definition of $\approx$, and as an immediate consequence, the maps $\mathscr{P}(\mathscr{M}) \rightarrow(\mathscr{P}(\mathscr{M}) / \sim)$ and $\mathscr{M}_{+} \rightarrow$ $\left(\mathscr{M}_{+} / \approx\right)$ are completely additive. In light of the factorizations (2.6) and (4.6), it is of course possible to transport these sum operations to $D(\mathscr{P}(\mathscr{M}))$ and $\widehat{T}\left(\mathscr{M}_{+}\right)$.

Pointwise lattice operations on pairs in $D(\mathscr{P}(\mathscr{M}))$ match (2.3) and so agree with the operations in $(\mathscr{P}(\mathscr{M}) / \sim)$, but meets and joins of infinite sets of continuous functions need not be continuous. For bounded real-valued functions on a stonean space, a regularization corrects this problem [33, Section III.1], but the situation for cardinal-valued functions is less clear.

Normality for $D$ and $\widehat{T}$ means appropriate analogues of (2.4). So how do we interpret an expression like "sup $D\left(p_{\alpha}\right)$ ", where $\left\{p_{\alpha}\right\}$ is an increasing net in $\mathscr{P}(\mathscr{M})$ ? As we just mentioned, the pointwise supremum need not lie in $D(\mathscr{P}(\mathscr{M}))$. Even when it does, Tomiyama showed that one may have $\sup D\left(p_{\alpha}\right) \neq D\left(\sup p_{\alpha}\right)$ [34, Example 1].

In the next section we show that $\sup \left[p_{\alpha}\right]$ always exists in $(\mathscr{P}(\mathscr{M}) / \sim)$. But the quotient map $\mathscr{P}(\mathscr{M}) \rightarrow(\mathscr{P}(\mathscr{M}) / \sim)$ still need not be normal; this can be seen from Lemma 3.2(2). For $p$ a properly infinite projection, the members of $[p]$, under the operator ordering, form an increasing net which converges strongly to $c(p)$. One obtains a counterexample to normality whenever $c(p) \notin$ $[p]$, and such counterexamples exist when $\kappa_{\mathscr{M}}>\aleph_{0}$. On the other hand, if $\kappa_{\mathcal{M}} \leq \aleph_{0}$, the quotient maps are given by the extended center-valued trace, which we know to be normal. We conclude

Proposition 5.1. Another equivalent condition in Proposition 3.8 is

(6) The quotient maps $\mathscr{P}(\mathscr{M}) \rightarrow(\mathscr{P}(\mathscr{M}) / \sim)$ and $\mathcal{M}_{+} \rightarrow\left(\mathcal{M}_{+} / \approx\right)$ are normal.

In contrast, a pointwise criterion for normality of $D$ and $\widehat{T}$ holds if and only if $\kappa_{\mathscr{M}} \leq \aleph_{0}$ and the center of $\mathcal{M}$ is finite-dimensional. We do not bother to prove this explicitly, but we mention an example. Let $\mathscr{M}=\ell^{\infty}$, and take $p_{n}$ to be the sum of the first $n$ elements of the standard basis. Since sup $D\left(p_{n}\right)$ does not agree with $D\left(\sup p_{n}\right)$ at any point of $(\beta \mathrm{N} \backslash \mathrm{N}) \subset \beta \mathrm{N} \simeq \Omega(\mathscr{Z}(\mathscr{M}))$, pointwise normality fails. And here $D$ is the identity - only the definition of "sup" has changed.

Our conclusion from all this is that the pointwise addition and lattice operations on functions in the range of $D$ and $\widehat{T}$ should be shelved in favor of the induced quotient structures on $(\mathscr{P}(\mathscr{M}) / \sim)$ and $\left(\mathscr{M}_{+} / \approx\right)$. With this interpret- 
ation the assertion " $D$ and $\widehat{T}$ are normal" is also equivalent to the conditions in Proposition 3.8.

\section{6. $(\mathscr{P}(\mathscr{M}) / \sim)$ is a complete lattice}

Having just been warned about the degeneracies of the pointwise ordering, we omit the last step of Tomiyama's construction for $D$ and stick with a more algebraic language. We follow the right-hand side of (2.5), further dividing $T\left(z^{f} p\right)$ into the pieces where it lies between consecutive finite cardinals. This allows us to write the typical element of $\widehat{T}\left(\mathscr{M}_{+}\right)$in the form

$$
\sum_{\kappa \leq \kappa, M} g_{\kappa} z_{\kappa}
$$

The meaning of this expression is as follows. If $\kappa$ is an infinite cardinal, then $g_{\kappa}=\kappa$. If $\kappa$ is a nonnegative integer, $g_{\kappa}$ is an element of $\mathscr{Z}(\mathscr{M})_{+}$satisfying $(\kappa-1) z_{\kappa} \leq g_{\kappa} \leq \kappa z_{\kappa}$ and $c\left(g_{\kappa}-(\kappa-1) z_{\kappa}\right)=z_{\kappa}$. The central projections $z_{\kappa}$ sum to 1 , and the decomposition is unique.

The partial order, pairwise sum operation, and pairwise lattice operations are easily understood for expressions of the form (6.1), but not all these belong to $\widehat{T}\left(\mathscr{M}_{+}\right)$. Consider the following conditions on an expression (6.1):

(a) taking no finite nonzero values on the type III summand;

(b) being less than or equal to $D\left(1_{\mathcal{M}}\right)$ (which does not depend on the choice of $D$ );

(b') being less than or equal to a finite multiple of $D\left(1_{\mathscr{M}}\right)$;

(c) having $g_{\kappa}=\kappa z_{\kappa}$ for finite $\kappa$ when restricted to the type I summand.

From Theorem 3.3 (plus the comments thereafter), Proposition 4.3, and the construction of $D$ in terms of $\kappa$-homogeneous projections, we deduce

- $\widehat{T}\left(\mathscr{M}_{+}\right)$is characterized by (a) and (b');

- $\widehat{T}\left(\mathscr{M}_{1}^{+}\right)$is characterized by (a) and (b);

- $D(\mathscr{P}(\mathscr{M}))$ is characterized by (a), (b), and (c).

Recall that a lattice is conditionally complete if one can take meets and joins of arbitrary bounded subsets.

THEOREM 6.1 .

(1) $(\mathscr{P}(\mathscr{M}) / \sim)$ and $\left(\mathscr{M}_{1}^{+} / \approx\right)$ are complete lattices.

(2) $\left(\mathscr{M}_{+} / \approx\right)$ is a conditionally complete lattice.

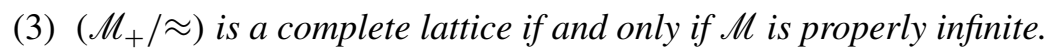


Proof. (1) We show how to perform lattice operations on expressions of the form (6.1). Our constructions preserve conditions (a), (b), and (c) above, so they are well-defined in $(\mathscr{P}(\mathscr{M}) / \sim)$ and $\left(\mathscr{M}_{1}^{+} / \approx\right)$.

Let us find the supremum of an arbitrary set $\left\{f^{\alpha}\right\}$, where

$$
f^{\alpha}=\sum g_{\kappa}^{\alpha} z_{\kappa}^{\alpha} .
$$

For each cardinal $\kappa \leq \kappa_{\mathcal{M}}$, set

$$
y_{\leq \kappa}=\bigwedge_{\alpha}\left(\sum_{\lambda \leq \kappa} z_{\lambda}^{\alpha}\right)
$$

$y_{\leq \kappa}$ is "where all $f^{\alpha}$ are $\leq \kappa$ ". Note that $y_{\leq \kappa}$ is increasing in $\kappa$ and $y_{\leq \kappa_{\mathcal{M}}}=1$. Next define, for each cardinal $\kappa \leq \kappa_{\mathscr{M}}$,

$$
z_{\kappa}=y_{\leq \kappa}-\bigvee_{\lambda<\kappa} y_{\leq \lambda} .
$$

The $z_{\kappa}$ are pairwise disjoint: if $\kappa_{1}<\kappa_{2}$, then

$$
z_{\kappa_{1}} \leq y_{\leq \kappa_{1}} \perp z_{\kappa_{2}} \text {. }
$$

Notice also that $\sum z_{\kappa}=1$. For if there were $z \in \mathscr{P}(\mathscr{Z}(\mathscr{M}))$ with $z \perp\left(\sum z_{\kappa}\right)$, then let $\lambda$ be the least cardinal with $z y_{\leq \lambda} \neq 0$; by definition $z z_{\lambda} \neq 0$ as well, which contradicts the assumption.

We claim that

$$
\sup _{\alpha} f^{\alpha}=\sum g_{\kappa} z_{\kappa} \triangleq f
$$

where $g_{\kappa}=\kappa$ when $\kappa$ is infinite, and otherwise $g_{\kappa}=\sup _{\alpha}\left(g_{\kappa}^{\alpha} z_{\kappa}\right)$, which exists as the supremum of a bounded set in $\mathscr{Z}(\mathscr{M})_{+}$.

Let us show that $f \geq f^{\alpha}$ for any $\alpha$. Fixing a cardinal $\lambda \leq \kappa_{\mathscr{M}}$,

$$
z_{\lambda} f^{\alpha}=z_{\lambda}\left(\sum g_{\kappa}^{\alpha} z_{\kappa}^{\alpha}\right)=\left(z_{\lambda} y_{\leq \lambda}\right)\left(\sum g_{\kappa}^{\alpha} z_{\kappa}^{\alpha}\right) \leq z_{\lambda}\left(\sum_{\kappa \leq \lambda} g_{\kappa}^{\alpha} z_{\kappa}^{\alpha}\right) .
$$

When $\lambda$ is infinite, we continue (6.3) as

$$
\leq \lambda z_{\lambda}=z_{\lambda} f
$$

When $\lambda$ is finite, we continue (6.3) as

$$
\leq z_{\lambda}(\lambda-1)\left(\sum_{\kappa<\lambda} z_{\kappa}^{\alpha}\right)+z_{\lambda} g_{\lambda}^{\alpha} z_{\lambda}^{\alpha} \leq z_{\lambda} g_{\lambda}=z_{\lambda} f .
$$


Since $z_{\lambda} f^{\alpha} \leq z_{\lambda} f$ for all $\lambda, f \geq f^{\alpha}$.

Finally we check that if $h=\sum h_{\kappa} x_{\kappa}$ satisfies $h \geq f^{\alpha}, \forall \alpha$, then necessarily $h \geq f$. Fixing a cardinal $\lambda \leq \kappa_{\mathcal{M}}$,

$$
\begin{aligned}
h_{\lambda} x_{\lambda}=x_{\lambda} h \geq x_{\lambda} f^{\alpha}, \forall \alpha & \Rightarrow x_{\lambda} \leq \sum_{\kappa \leq \lambda} z_{\kappa}^{\alpha}, \forall \alpha \\
& \Rightarrow x_{\lambda} \leq \bigwedge_{\alpha}\left(\sum_{\kappa \leq \lambda} z_{\kappa}^{\alpha}\right)=y_{\leq \lambda} .
\end{aligned}
$$

This last inequality implies

$$
x_{\lambda} f=x_{\lambda} y_{\leq \lambda} f \leq x_{\lambda}\left(\sum_{\kappa \leq \lambda} g_{\kappa} z_{\kappa}\right) .
$$

When $\lambda$ is infinite, we continue (6.4) as

$$
\leq \lambda x_{\lambda}=x_{\lambda} h .
$$

When $\lambda$ is finite, we continue (6.4) as

$$
\leq x_{\lambda}(\lambda-1)\left(\sum_{\kappa<\lambda} z_{\kappa}\right)+x_{\lambda} g_{\lambda} z_{\lambda}
$$

and the inequality $h \geq f^{\alpha}, \forall \alpha$, allows us to compute further

$$
=x_{\lambda}(\lambda-1)\left(\sum_{\kappa<\lambda} z_{\kappa}\right)+x_{\lambda}\left(\sup _{\alpha} g_{\lambda}^{\alpha} z_{\lambda}\right) \leq x_{\lambda} h .
$$

Since $x_{\lambda} f \leq x_{\lambda} h$ for all $\lambda, f \leq h$.

This completes the proof that $f=\sup f^{\alpha}$.

As for the infimum of the $f^{\alpha}$, we first point out that we cannot write anything like

$$
\bigwedge f^{\alpha}=1-\left(\bigvee\left(1-f^{\alpha}\right)\right)
$$

which is a useful duality in $\mathscr{P}(\mathscr{M})$. There is no complementation in the lattices $(\mathscr{P}(\mathscr{M}) / \sim)$ and $\left(\mathscr{M}_{1}^{+} / \approx\right)$, at least when $\mathscr{M}$ is not finite. Instead we define

$$
y_{\leq \kappa}=\bigvee_{\alpha}\left(\sum_{\lambda \leq \kappa} z_{\lambda}^{\alpha}\right)
$$

and complete the rest of the proof similarly to the proof for the supremum. (The substitute for (6.3) should begin with " $z_{\lambda}^{\alpha} f=\ldots$ ”, for (6.4) should begin with " $z_{\lambda} h=\ldots$..) 
(2) If a family in $\widehat{T}\left(\mathscr{M}_{+}\right)$has an upper bound in $\widehat{T}\left(\mathscr{M}_{+}\right)$, then apparently the supremum of the corresponding expressions (6.1) satisfies (b'), so belongs to $\widehat{T}\left(\mathscr{M}_{+}\right)$. Any family in $\widehat{T}\left(\mathscr{M}_{+}\right)$is bounded below by 0 and has an infimum.

(3) When $\mathcal{M}$ is properly infinite, any finite multiple of $D\left(1_{\mathscr{M}}\right)$ is again equal to $D\left(1_{\mathscr{M}}\right)$, so conditions (b) and (b') agree. If $\mathcal{M}$ has a finite summand $z \mathscr{M}$, the elements $\widehat{T}(n z)=n z$ have supremum $\aleph_{0} z \notin \widehat{T}\left(\mathscr{M}_{+}\right)$.

There should be no confusion between Theorem 6.1(2) and the conditionally complete lattice structure that $\mathscr{M}_{+}$acquires from the spectral order [28].

\section{Application to representation theory}

In this section we reinterpret Theorem 6.1 in terms of the (nondegenerate normal Hilbert space) representations of $\mathscr{M}$. Unless noted otherwise, we use "isomorphism" in the sense of normed $\mathscr{M}$-modules, i.e.

$$
\begin{gathered}
\left\{\pi_{1}, \mathfrak{S}_{1}\right\} \simeq\left\{\pi_{2}, \mathfrak{S}_{2}\right\} \Longleftrightarrow \\
\exists \text { unitary } U: \mathfrak{S}_{1} \rightarrow \mathfrak{S}_{2}: \quad U \pi_{1}(x) U^{*}=\pi_{2}(x), \quad \forall x \in \mathcal{M} .
\end{gathered}
$$

It follows from the basic theory (see [15, Sections 2.1-2] or [31, Section 2]) that any representation is (isomorphically) contained in a direct sum of copies of the standard form $\left\{\mathrm{id}, L^{2}(\mathscr{M})\right\}$. We view $\oplus_{I} L^{2}(\mathscr{M})$ as a row vector and think of the $\mathscr{M}$-action as multiplication on the left. The commutant is right multiplication by $\mathscr{B}\left(\ell_{I}^{2}\right) \bar{\otimes} \mathcal{M}$, and the closed submodules are of the form $\left(\oplus_{I} L^{2}(\mathscr{M})\right) q$, where $q \in \mathscr{P}\left(\mathscr{B}\left(\ell_{I}^{2}\right) \bar{\otimes} \mathscr{M}\right)$. Two submodules are isomorphic if and only if the corresponding projections are equivalent.

This means that the isomorphism class of a representation corresponds to an equivalence class of projections in some amplification of $\mathscr{M}$. Adding representations corresponds to adding equivalence classes. As we have mentioned, the partial order can be defined in terms of the sum, so provided we make some kind of size restriction, we get an isomorphism of ordered monoids. For example, if $\mathcal{M}$ is $\sigma$-finite, we obtain an identification between $\left(\mathscr{P}\left(\mathscr{B}\left(\ell^{2}\right) \bar{\otimes} \mathscr{M}\right) / \sim\right)$ and isomorphism classes of countably generated Hilbert $\mathscr{M}$-modules. This all works for $L^{p}$ modules [16], too, and is closely related to the $K_{0}$ functor [14], [35].

(Most of the ideas of the preceding two paragraphs were discussed by Breuer [1], [2], without making reference to standard forms. He focused on the monoid generated by equivalence classes of finite projections, because the associated Grothendieck group, called the index group of $\mathcal{M}$, is the natural carrier for the Fredholm theory of $\mathcal{M}$. Olsen [27] later combined Breuer's work with Tomiyama's dimension function to give a very general version of index theory in von Neumann algebras.) 
CoROLlaRY 7.1. Let $\left\{\pi_{\alpha}, \mathfrak{S}_{\alpha}\right\}$ be a set of representations of a fixed von Neumann algebra $\mathscr{M}$. Then there is a maximal representation of $\mathscr{M}$ which is (isomorphically) contained in all of these, and there is a minimal representation which (isomorphically) contains all of these. Both are unique up to $M$-module isomorphism.

PRoof. Choose a large enough set $I$ so that for all $\alpha,\left\{\pi_{\alpha}, \mathfrak{S}_{\alpha}\right\}$ is a subrepresentation of $\left\{\mathrm{id}, \oplus_{I} L^{2}(\mathscr{M})\right\}$. The corollary follows from the preceding discussion and the fact that $\left(\mathscr{P}\left(\mathscr{B}\left(\ell_{I}^{2}\right) \bar{\otimes} \mathscr{M}\right) / \sim\right)$ is a complete lattice.

In the early years of the subject, von Neumann algebras were generally tied to their Hilbert spaces, and the notion of $\mathscr{M}$-module isomorphism was therefore not in use. Instead, one classified represented algebras up to the slightly weaker notion of spatial isomorphism, which allows for an arbitrary isomorphism between the algebras. (An $\mathscr{M}$-module isomorphism between representations $\left\{\pi_{1}, \mathfrak{F}_{1}\right\}$ and $\left\{\pi_{2}, \mathfrak{F}_{2}\right\}$ is a spatial isomorphism between von Neumann algebras $\left\{\pi_{1}(\mathscr{M}), \mathfrak{F}_{1}\right\}$ and $\left\{\pi_{2}(\mathscr{M}), \mathfrak{F}_{2}\right\}$ which induces the algebra isomorph$\operatorname{ism} \pi_{2} \circ \pi_{1}^{-1}$.) The question "When is an algebraic isomorphism of represented von Neumann algebras spatial?", which is a noncommutative version of the fundamental problem of unitary equivalence for normal operators, is treated in detail in [18]. Also see [4] for a projection-based approach to the existence of spatial isomorphisms.

Having said that, equivalence classes of representations/represented algebras were first studied by Murray and von Neumann [25, Chapter III], using the coupling constant for finite factors. The generalizations to coupling functions and arbitrary algebras were the motivations for the Griffin and Pallu de la Barrière results featured in Section 4. The space-free approach was notably developed by the Japanese school of the 1950's.

Modulo spatial isomorphism, the set of equivalence classes of representations of a fixed von Neumann algebra may not even be partially ordered. We mention the relevant example. Let $\mathcal{M}$ be a type $\mathrm{II}_{\infty}$ factor with dimension function $D$ and fundamental group $\Gamma \notin\{\{1\},(0, \infty)\}$. (The existence of such an $\mathscr{M}$ remained in doubt until a breakthrough of Connes in 1980 [3]. The fundamental group of a $\mathrm{II}_{\infty}$ factor can be defined as

$$
\{\lambda \in(0, \infty) \mid \exists \alpha \in \operatorname{Aut}(\mathcal{M}), D \circ \alpha=\lambda D\},
$$

with the group operation being multiplication.) Kadison [17] showed that for nonzero finite projections $p, q, L^{2}(\mathscr{M}) p$ is spatially isomorphic to $L^{2}(\mathscr{M}) q$ if and only if $\frac{D(p)}{D(q)} \in \Gamma$. Since $\Gamma \neq(0, \infty)$, we may find nonzero finite projections $p, p^{\prime}$ with $\frac{D(p)}{D\left(p^{\prime}\right)} \notin \Gamma$. And $\Gamma \neq\{1\}$, so we may find spatial isomorphisms $L^{2}(\mathscr{M}) q_{1} \simeq L^{2}(\mathscr{M}) p^{\prime} \simeq L^{2}(\mathcal{M}) q_{2}$ with $q_{1} \supsetneqq p \supsetneqq q_{2}$. Therefore the spatial 
equivalence class of $L^{2}(\mathscr{M}) p$ both dominates and is dominated by that of $L^{2}(\mathscr{M}) p^{\prime}$, yet the two are not equal.

At least for factors, this kind of pairing $-\mathrm{II}_{\infty}$ algebra, $\mathrm{II}_{1}$ commutant - is the only case where the two notions of equivalence differ. Not coincidentally, the only choice required for $T, D$, and $\widehat{T}$ is the normalization on the finite elements in the $\mathrm{II}_{\infty}$ summand. (On a $\mathrm{II}_{\infty}$ summand, one possible definition for "normalization" is the inverse image of the identity, which is nothing but the equivalence class of the projection $p$ discussed in Remark 4.7.)

Acknowledgements. We thank Chuck Akemann and Ken Goodearl for many helpful conversations.

\section{REFERENCES}

1. Breuer, M., Fredholm theories in von Neumann algebras I, Math. Ann. 178 (1968), 243-254.

2. Breuer, M., Fredholm theories in von Neumann algebras II, Math. Ann. 180 (1969), 313-325.

3. Connes, A., A factor of type $\mathrm{II}_{1}$ with countable fundamental group, J. Operator Theory 4 (1980), 151-153.

4. Digernes, T., On classes of projections in a von-Neumann algebra, Math. Scand. 31 (1972), 191-200.

5. Dixmier, J., Les anneaux d'opérateurs de classe finie, Ann. Sci. École Norm. Sup. (3) 66 (1949), 209-261.

6. Dixmier, J., Sur certains espaces considérés par M. H. Stone, Summa Brasil. Math. 2 (1951), $151-182$.

7. Dixmier, J., Applications $\natural$ dans les anneaux d'opérateurs, Compositio Math. 10 (1952), $1-55$.

8. Dixmier, J., Les Algèbres d'Opérateurs dans l'Espace Hilbertien (Algèbres de von Neumann), Gauthier-Villars, Paris, 1969.

9. Fillmore, P., The dimension theory of certain cardinal algebras, Trans. Amer. Math. Soc. 117 (1965), 21-36.

10. Goodearl, K., and Wehrung, F., The complete dimension theory of partially ordered systems with equivalence and orthogonality, Mem. Amer. Math. Soc. 176 (2005), no. 831.

11. Griffin Jr., E. L., Some contributions to the theory of rings of operators, Trans. Amer. Math. Soc. 75 (1953), 471-504.

12. Griffin Jr., E. L., Some contributions to the theory of rings of operators II, Trans. Amer. Math. Soc. 79 (1955), 389-400.

13. Haagerup, U., Normal weights on $W^{*}$-algebras, J. Funct. Anal. 19 (1975), 302-317.

14. Handelman, D., $K_{0}$ of von Neumann and $A F C^{*}$ algebras, Quart. J. Math. Oxford Ser. (2) 29 (1978), 427-441.

15. Jones, V., and Sunder, V. S., Introduction to Subfactors, London Math. Soc. Lecture Note Ser. 234 (1997).

16. Junge, M., and Sherman, D., Noncommutative $L^{p}$ modules, J. Operator Theory 53 (2005), 3-34.

17. Kadison, R. V., Isomorphisms of factors of infinite type, Canad. J. Math. 7 (1955), 322-327.

18. Kadison, R. V., Unitary invariants for representations of operator algebras, Ann. of Math. (2) 66 (1957), 304-379.

19. Kadison, R. V., and Pedersen, G. K., Equivalence in operator algebras, Math. Scand. 27 (1970), 205-222. 
20. Kadison, R. V., and Ringrose, J. R., Fundamentals of the Theory of Operator Algebras I, Grad. Stud. Math. 15 (1997).

21. Kadison, R. V., and Ringrose, J. R., Fundamentals of the Theory of Operator Algebras II, Grad. Stud. Math. 16 (1997).

22. Loomis, L. H., The lattice theoretic background of the dimension theory of operator algebras, Mem. Amer. Math. Soc. (1955), no. 18.

23. Maeda, S., Dimension functions on certain general lattices, J. Sci. Hiroshima Univ. Ser. A. 19 (1955), 211-237.

24. Murray, F. J., and von Neumann, J., On rings of operators, Ann. of Math. (2) 37 (1936), $116-229$.

25. Murray, F. J., and von Neumann, J., On rings of operators IV, Ann. of Math. (2) 44 (1943), 716-808.

26. Nelson, E., Notes on non-commutative integration, J. Funct. Anal. 15 (1974), 103-116.

27. Olsen, C. L., Index theory in von Neumann algebras, Mem. Amer. Math. Soc. 47 (1984), no. 294.

28. Olson, M. P., The selfadjoint operators of a von Neumann algebra form a conditionally complete lattice, Proc. Amer. Math. Soc. 28 (1971), 537-544.

29. Pallu de la Barrière, R., Sur les algèbres d'opérateurs dans les espaces hilbertiens, Bull. Soc. Math. France 82 (1954), 1-52.

30. Ringrose, J. R., Linear mappings between operator algebras, in Sympos. Math. XX (Convegno sulle Algebre $C^{*}$ e loro Applicazioni in Fisica Teorica, Convegno sulla Teoria degli Operatori Indice e Teoria $K$, INDAM, Roma, 1975), pp. 297-315, Academic Press, London, 1976.

31. Sherman, D., Relative tensor products for modules over von Neumann algebras, in Function Spaces (Edwardsville, 2002), 275-291, Contemp. Math. 328 (2003).

32. Sherman, D., Unitary orbits of normal operators in von Neumann algebras, J. Reine Angew. Math., to appear.

33. Takesaki, M., Theory of Operator Algebras I, Springer-Verlag, Berlin, 1979.

34. Tomiyama, J., Generalized dimension function for $W^{*}$-algebras of infinite type, Tôhoku Math. J. (2) 10 (1958), 121-129.

35. Wegge-Olsen, N., K-Theory and $C^{*}$-Algebras, Oxford University Press, New York, 1993.

Department of Mathematics

University of California

Santa Barbara, CA 93106

USA

E-mail: dsherman@math.ucsb.edu 